\title{
Epidemiologia dos gliomas no estado do Pará - Parte I (2000-2006)
}

\author{
Edmundo Luís Rodrigues Pereira ${ }^{1}$, Daniella Brito Rodrigues², \\ Maria Luana Carvalho Viegas², Mário de Nazareth Hermes \\ Junior ${ }^{3}$, Rommel Mario Rodríguez Burbano ${ }^{4}$
}

\section{RESUMO}

Objetivo: O presente estudo tem como principal objetivo realizar uma análise epidemiológica inicial acerca dos tumores intracranianos primários no estado do Pará, com foco em gliomas, baseada nos registros hospitalares de um hospital estadual de referência em câncer (Hospital Ophir Loyola - HOL), durante os anos de 2000 a 2006. Métodos: Análise epidemiológica descritiva, retrospectiva e observacional dos prontuários de 457 pacientes submetidos a tratamento cirúrgico por tumores intracranianos primários (TIP) entre os anos de 2000 e 2006, no Hospital Ophir Loyola (HOL), tendo sido incluídos dados referentes a sexo, etnia, procedência, idade, escolaridade, estado civil, localização do tumor, tipo histológico, realização de quimioterapia e/ou radioterapia e ocorrência de óbito. Resultados: Os resultados mostraram taxa média de 1,22 tumor intracraniano para cada 100 mil habitantes, com predomínio de gliomas (70\%) e meningiomas (15\%). Ao se analisar o grupo de gliomas por subtipo histológico, encontrou-se predomínio de astrocitomas malignos (graus III e IV, OMS), sendo o glioblastoma o tipo histológico mais encontrado (32\% do total e $45 \%$ dos gliomas, respectivamente). Conclusão: Os resultados encontrados sugerem discrepâncias relacionadas a dificuldades regionais de acesso ao atendimento especializado e com a notificação e registro dos pacientes com diagnóstico de TIP, fornecendo dados relevantes para caracterizar esse grupo de neoplasias em termos populacionais, permitindo intervenções por parte das autoridades responsáveis.

\section{PALAVRAS-CHAVE}

Glioma, neoplasias, epidemiologia.

\section{ABSTRACT}

Epidemiology of gliomas in Pará province, Brazil - Part I (2000-2006)

Objective: The present study has as its main objective to perform an epidemiological analysis about the primary intracranial tumors in the state of Pará, focusing on gliomas, based on hospital records of the state hospital in reference cancer (Ophir Loyola Hospital - HOL) during the years 2000 to 2006. Methods: Epidemiological study descriptive, retrospective and observational of medical records of 457 patients who underwent surgical treatment for intracranial tumors primary (TIP) between the years 2000 and 2006 in Ophir Loyola Hospital (HOL), it was included data on gender, ethnicity, origin, age, education, marital status, tumor location, histological type, undergo chemotherapy and/or radiotherapy and occurrence of death. Results: The results showed an average rate of 1.22 intracranial tumors per 100,000 population, with a prevalence of gliomas (70\%) and meningiomas (15\%). When analyzing the group of gliomas by histological subtype, found predominance of malignant astrocytomas (grades III and IV, WHO), and the glioblastoma histological type was the most commonly found (32\% of the total and $45 \%$ of gliomas, respectively). Conclusion: The results suggest discrepancies related to regional difficulties of access to specialized care and the notification and registration of patients diagnosed with TIP, providing relevant data to characterize this group of neoplasms in population terms and allowing intervention by the authorities.

\section{KEYWORDS}

Glioma, neoplasms, epidemiology.

1 Neurocirurgião da Clínica Neurológica do Hospital Estadual Ophir Loyola, professor da disciplina de Neurologia da Universidade Federal do Pará (UFPA), Belém, PA, Brasil.

2 Acadêmica de Medicina da Universidade do Estado do Pará (UEPA) e da UFPA, Belém, PA, Brasil.

3 Neurocirurgião da Clínica Neurológica do Hospital Estadual Ophir Loyola, Belém, PA, Brasil.

4 Doutor em Genética pela Universidade de São Paulo (USP), SP, Brasil. Hospital Ophir Loyola (HOL), Belém, PA, Brasil. 


\section{Introdução}

Tumores intracranianos primários (TIP) são aqueles que se originam de células e estruturas localizadas no interior do crânio - neurônios, células da glia, membrana aracnoide, glândula pituitária, entre outras. São responsáveis por aproximadamente $1,5 \%$ de todos os cânceres humanos e por $2,4 \%$ de todas as mortes decorrentes de neoplasias, todos os anos. ${ }^{1-3}$

Conforme o relato da Central Americana de Registro de Tumores Cerebrais (CBTRUS - Central Brain Tumor Registry of the United States, 2010), TIP constituem a segunda causa de morte por câncer abaixo dos 15 anos de idade e a terceira causa de morte por câncer abaixo dos 30 anos. $^{2}$ Os tumores localizados acima da tenda do cerebelo (supratentoriais) são os mais frequentes em adultos, dentre os quais os gliomas (astrocitomas, ependimomas, oligodendrogliomas) são responsáveis por mais de 30\% dos tumores nesse grupo. Por outro lado, em crianças, a localização mais comum é infratentorial, isto é, na fossa posterior, sendo os tipos mais frequentes os meduloblastomas, ependimomas e astrocitomas pilocíticos do cerebelo. ${ }^{1,3-5}$

O termo "glioma" é utilizado para denominar os tumores que se originam nas células da glia - astrocitomas, oligodendrogliomas e ependimomas. Segundo a classificação da Organização Mundial de Saúde (OMS), são separados em graus crescentes, de acordo com a presença de sinais de malignidade, em I, II, III (astrocitoma anaplásico) e IV (glioblastoma ou GBM). ${ }^{6-11}$ Esse grupo de tumores primários é o mais frequente no adulto, ${ }^{4,711-17}$ e, entre esses, a forma mais comum é também a mais maligna e incurável (glioblastoma astrocitoma grau IV). ${ }^{16-19}$

Segundo dados do Instituto Nacional de Câncer (INCA), os tumores primários do SNC possuem distribuição regional bastante heterogênea no Brasil, ocupando, em homens, a oitava colocação na região Centro-Oeste (6/100 mil), com equivalência nas regiões Sul (7/100 mil) e Nordeste (3/100 mil) (nona posição), enquanto nas regiões Sudeste $(6 / 100$ mil) e Norte (2/100 mil) se encontram na décima posição. No caso das mulheres, tal distribuição se repete, sendo o oitavo tipo de câncer mais frequente nas regiões Sul (6/100 mil) e Centro-Oeste $(4 / 100 \mathrm{mil})$, com equivalência entre as regiões Norte (2/100 mil, décima colocação) e regiões Sudeste (5/100 mil) e Nordeste (3/100 mil, décima-primeira colocação). Na região Norte, o estado de Roraima possui a maior incidência (3,71 casos para cada 100 mil habitantes), seguido pelo Amazonas (3,09/100 mil), Rondônia (3,01/100 mil), Maranhão (2,08/100 mil), Amapá (1,83/100 mil) e Pará (1,20/100 mil). ${ }^{6}$ A expectativa para o ano de 2012 no Brasil é de que haverá 4.820 casos novos em homens e $4.450 \mathrm{em}$ mulheres, correspondendo a um risco estimado de cinco casos novos a cada 100 mil homens e quatro casos novos a cada 100 mil mulheres. ${ }^{6}$

Os resultados do presente estudo, ainda que próximos do esperado, mostram algumas discrepâncias que traduzem os grandes déficits de desenvolvimento em algumas regiões do Brasil, como é o caso da região Norte. ${ }^{6,20-23}$ No Pará, grandes distâncias, combinadas com escassez de hospitais, contribuem para a dificuldade de acesso dos pacientes à atenção médica, culminando em registros deficitários. $\mathrm{O}$ presente estudo tem como principal objetivo realizar uma análise epidemiológica inicial acerca dos tumores intracranianos primários no estado do Pará, com foco em gliomas, baseada nos registros hospitalares do Hospital Estadual de referência em câncer (Hospital Ophir Loyola - HOL), durante os anos de 2000 a 2006, esperando com isso contribuir para minimizar o déficit de informação e para a melhor caracterização da doença no estado e na região Norte do país.

\section{Metodologia}

A presente pesquisa realizou estudo transversal, retrospectivo, do tipo observacional, com análise da incidência de tumores intracranianos primários em adultos durante o período de 2000 a 2006, com enfoque epidemiológico nos gliomas tratados no hospital de referência em câncer, no estado do Pará (Hospital Ophir Loyola).

Para tanto, adotaram-se como base informações colhidas no setor de registro hospitalar do serviço de arquivos e prontuários da instituição. Foram incluídos os dados referentes ao período citado (julho de 2000 a julho de 2006), constando, entre outros, sexo, etnia, procedência, idade, escolaridade, estado civil, localização do tumor, tipo histológico, realização de quimioterapia e/ou radioterapia e ocorrência de óbito. Foram excluídos dados incompletos e/ou inconclusivos, assim como algumas neoplasias mais raras com pouca significância epidemiológica. Nessa primeira parte do estudo, procurou-se fixar a atenção naquelas neoplasias de maior frequência segundo a literatura especializada. ${ }^{2,3,6,11,12,24-26}$ Para tal, foram disponibilizados os dados de 457 pacientes com o diagnóstico genérico de "Tumores Intracranianos" (CID-10: C 71 D 43, D 32, D 33). Após avaliação dos registros clínicos e resultados de histologia, foram selecionados 203 pacientes, que foram reagrupados segundo tipo histológico, topografia, dados individuais (sexo, idade, procedência, profissão), período de internação, ocorrência de óbito e tipo de tratamento (cirurgia, radioterapia e quimioterapia). Dos 203 casos de TIP, quatro foram excluídos, por apresen- 
tarem incongruências entre os antecedentes clínicos e o diagnóstico histológico fornecido.

As diversas variáveis analisadas foram organizadas em banco de dados tabulado para posterior processamento nos softwares Microsoft Office Excel 2007 e Bioestat 5.0, com a confecção de gráficos e tabelas para organização e sistematização dos dados e suas conclusões.

\section{Resultados}

No período compreendido entre julho de 2000 e julho de 2006, foram tratados cirurgicamente no HOL 203 pacientes cujo resultado histopatológico confirmou tratar-se de neoplasia primária do cérebro (Tabela 1), notando-se incidência crescente a partir do ano 2000 e pico em 2003 e 2005 (18\%), com a menor incidência no ano de 2000 (8\%) (Figura 1). Quatro pacientes foram excluídos por causa da incongruência entre os antecedentes clínicos de neoplasia conhecida e previamente tratada em outros órgãos (três adenocarcinomas de mama e um carcinoma de broncogênico) com o diagnóstico histopatológico de "glioblastoma" e cujos exames de imagem (TC/RM) indicavam, na verdade, neoplasias extra-axiais sugestivas de metástases.

Quanto ao sexo, houve discreto predomínio do sexo masculino (52\%) em relação ao feminino (48\%), sendo a idade média dos homens igual a 36,54 e a das mulheres, 36,97.

Gliomas representam 70\% da amostra, com incidência crescente a partir do ano de 2000, com pico em 2006 (Figura 1). Nos demais tipos histológicos, meningiomas predominam com 15\% do total de casos (Figura 2).

Considerando somente o grupo dos gliomas, o glioblastoma foi o subtipo mais frequente (32\%), seguido por astrocitoma benigno (21\%), perfazendo, ambos, $53 \%$ de todos os TIP operados na instituição, seguidos por astrocitomas anaplásicos $(6,5 \%)$, ependimomas $(6,5 \%)$, oligodendrogliomas $(3 \%)$ e gliomas mistos (1\%) (Figura 2).

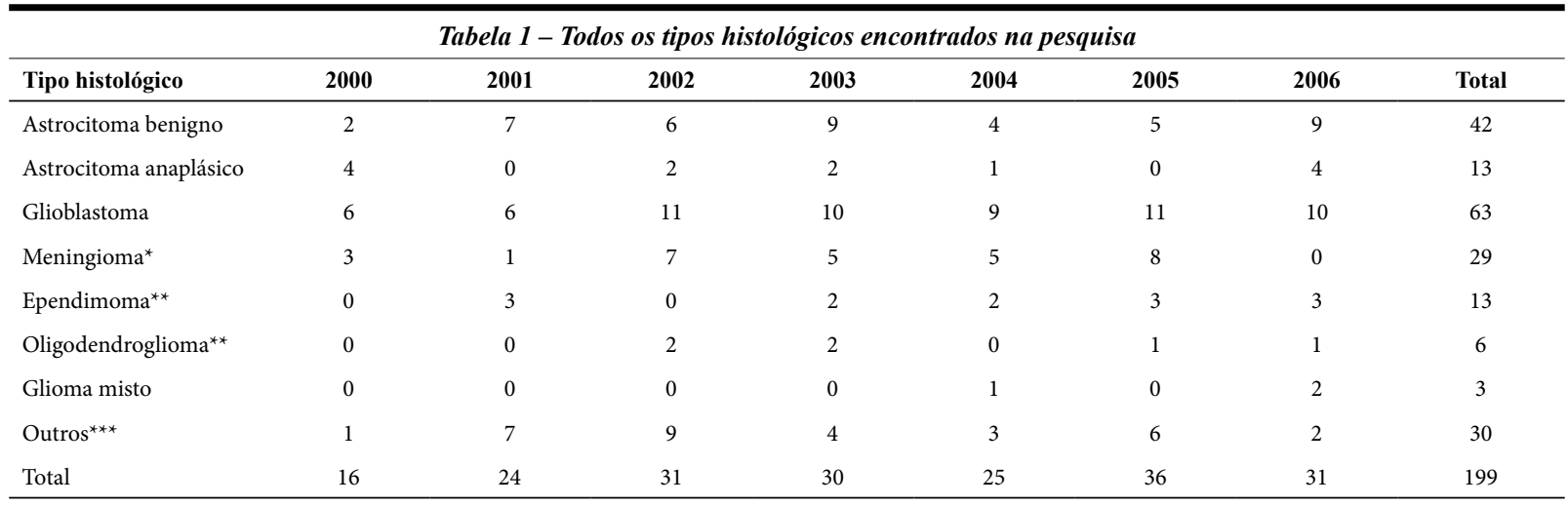

* Meningiomas malignos: 2 .

** Ependimomas, SOE, Oligodendroglioma, SOE.

*** Outros: cordoma, linfoma, tumor neuroectodérmico primitivo (supratentorial), meduloepitelioma, tumores da região hipofisária, tumores da região pineal, tumor disembrioplásico primitivo (DNET).

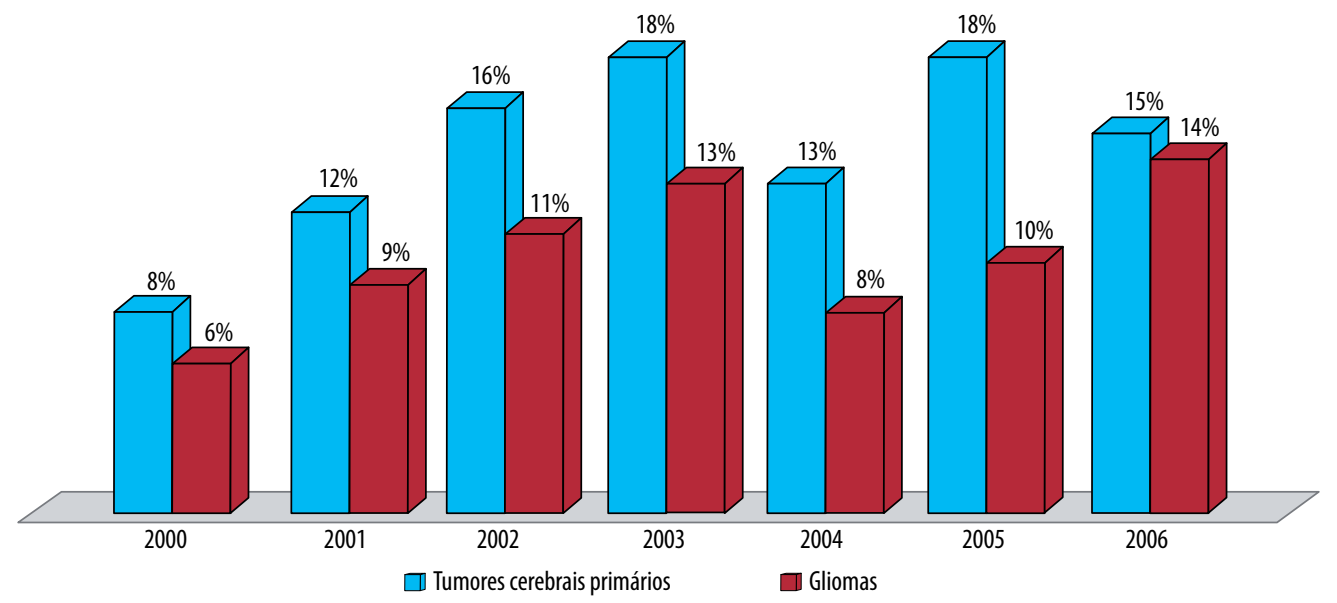

Figura 1 - Relação entre a incidência total de tumores cerebrais primários e gliomas de acordo com os anos analisados. 


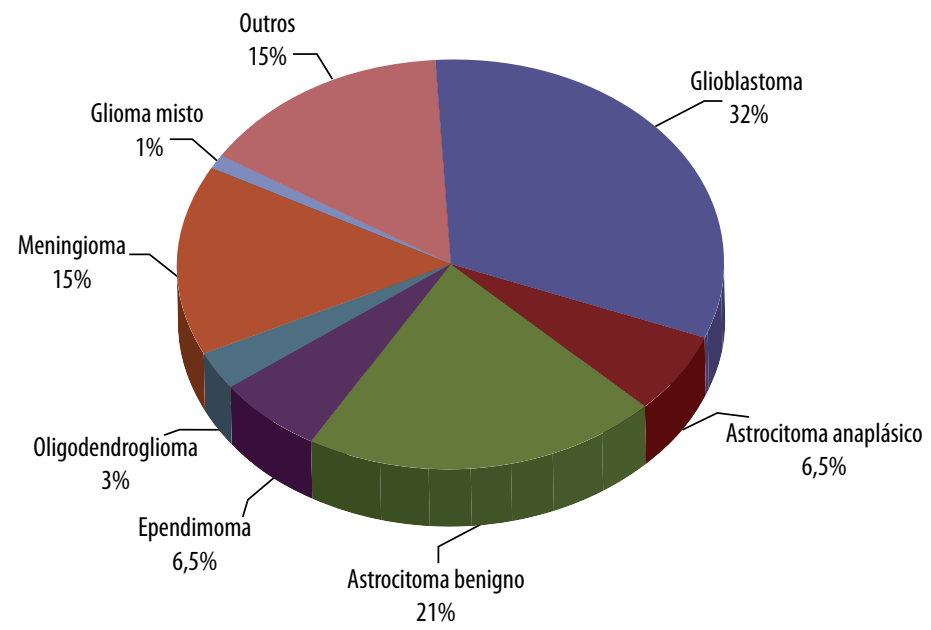

Figura 2 - Todos os TIP, de acordo com histologia. Nota-se predomínio dos gliomas (70\% da amostra).

Avaliando separadamente o grupo dos gliomas, astrocitomas correspondem a $84,5 \%$ da amostra, predominando glioblastomas (45\%), seguidos pelos astrocitomas benignos com $30 \%$ e pelos astrocitomas anaplásicos (9,5\%); entre os demais gliomas, predominaram os ependimomas com 9,5\% (Figura 3). Em conjunto, as- trocitomas malignos (graus III e IV, OMS) representam mais da metade $(54,5 \%)$ do total de gliomas.

Astrocitomas "benignos" (graus I e II, OMS) perfazem 36\% da amostra com distribuição uniforme entre astrocitomas fibrilares (7\%), astrocitomas pilocíticos (4\%) e xantoastrocitomas pleomórficos (2\%) (Figura 4).

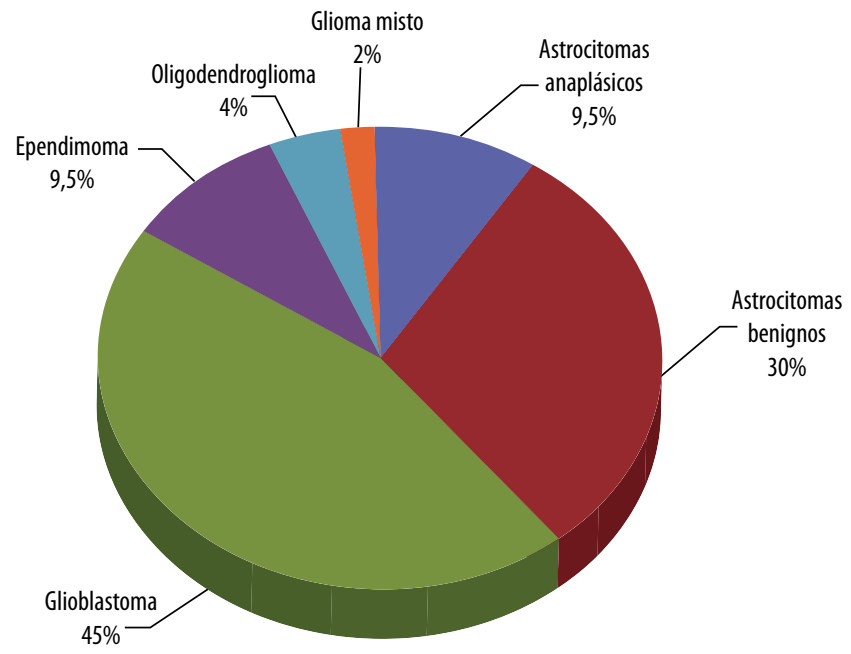

Figura 3 - Distribuição total da incidência de gliomas. Predominam astrocitomas (84,5\%) do total de gliomas.

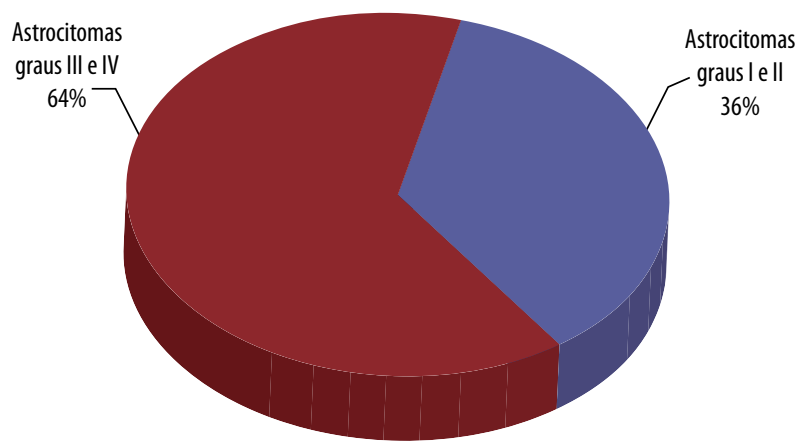

Figura 4 - Relação entre astrocitomas malignos (graus III e IV) e astrocitomas graus I e II. 


\section{Discussão}

Há poucos trabalhos na literatura brasileira abordando especificamente o tema "epidemiologia de gliomas". Tal deficiência talvez seja um reflexo da falta de centros regionais com objetivo específico de registrar os casos diagnosticados e tratados nos hospitais que prestam atendimento ao Sistema Único de Saúde (SUS) em tumores do SNC. Esse déficit de informação certamente dificulta a adoção de medidas e ações preventivas e de intervenção, por parte das secretarias de saúde. ${ }^{6,9,27}$ As variações numéricas observadas no presente estudo refletem também as grandes diferenças no desenvolvimento de cada região, podendo inclusive refletir uma incidência distinta da real, mesmo se considerando que a amostra analisada é proveniente do único hospital estadual que se propõe a tratar câncer, em todo o estado do Pará, cujas grandes distâncias e a dificuldade de acesso dos pacientes à atenção médica devem acarretar vieses de registro, explicando, desse modo, algumas discrepâncias observadas entre as incidências globais de TIP encontradas na região Norte e aquelas encontradas nas regiões Sul e Sudeste (Figuras 5 e 6). Mesmo assim, o presente estudo fornece dados bastante aproximados da realidade assistencial em nosso meio, ressaltando-se ainda que a maioria dos enfermos é proveniente da própria cidade de Belém e de seu entorno, com uma minoria proveniente de cidades distantes e isoladas no interior do estado, como é o caso, por exemplo, dos pacientes oriundos de cidades distantes do interior do estado, desprovidas de meios terrestres adequados de acesso até Belém, com deslocamentos que duram algumas vezes até 12 horas.

A incidência total média de TIP estimada na presente pesquisa foi de 1,22/100 mil habitantes, e a incidência total de gliomas foi de 0,37/100 mil habitantes. Essa incidência de neoplasias cerebrais é significativamente inferior ao trabalho de Parkin et $a .^{23}$, que, ao analisar TIP em todo o mundo, encontrou uma incidência de 3,7/100 mil habitantes entre os homens e de 2,6/100 mil habitantes entre as mulheres, divergindo também de Gomes ${ }^{31}$ e de outros mais recentes, como Kohler et al. ${ }^{25}$, Samuels ${ }^{1}$ e da própria Central Americana de Registro de Tumores Cerebrais (CBTRUS), para o ano de $2012^{2}$ (19.89/100 mil habitantes). É importante ressaltar que a incidência encontrada no presente estudo, apesar de oriunda dos anos de 2000-2006, é concordante com as estimativas de câncer do SNC encontradas pelo INCA para o ano de 2012 (1,20/100 mil habitantes $)^{6}$ e inferior a dos demais estados da região Norte (Figura 6).

Ao se analisar a incidência de gliomas isoladamente, observa-se similar discrepância em relação aos dados norte-americanos relatados pela CBTRUS, ${ }^{2}$ em que os gliomas foram responsáveis por aproximadamente 6.04 dos casos de tumores cerebrais para cada $100 \mathrm{mil}$ habitantes. Essa divergência pode ser entendida pelas limitações associadas ao desenvolvimento da região Norte como um todo, com dificuldade de acesso da população aos escassos serviços de saúde pública especializada, distribuídos nas enormes distâncias do estado, onde muitas localidades são destituídas de vias de acesso até a cidade de Belém.

A predominância dos gliomas na amostra (70\% do total de tumores cerebrais) é significativamente superior quando comparada à relatada pela CBTRUS $(30 \%$ dos tumores cerebrais primários), ${ }^{1,2,5,11,24,27-30}$ porém são números similares aos relatados por Schwartzbaum et al. ${ }^{4}$ em 2006 (77\%).

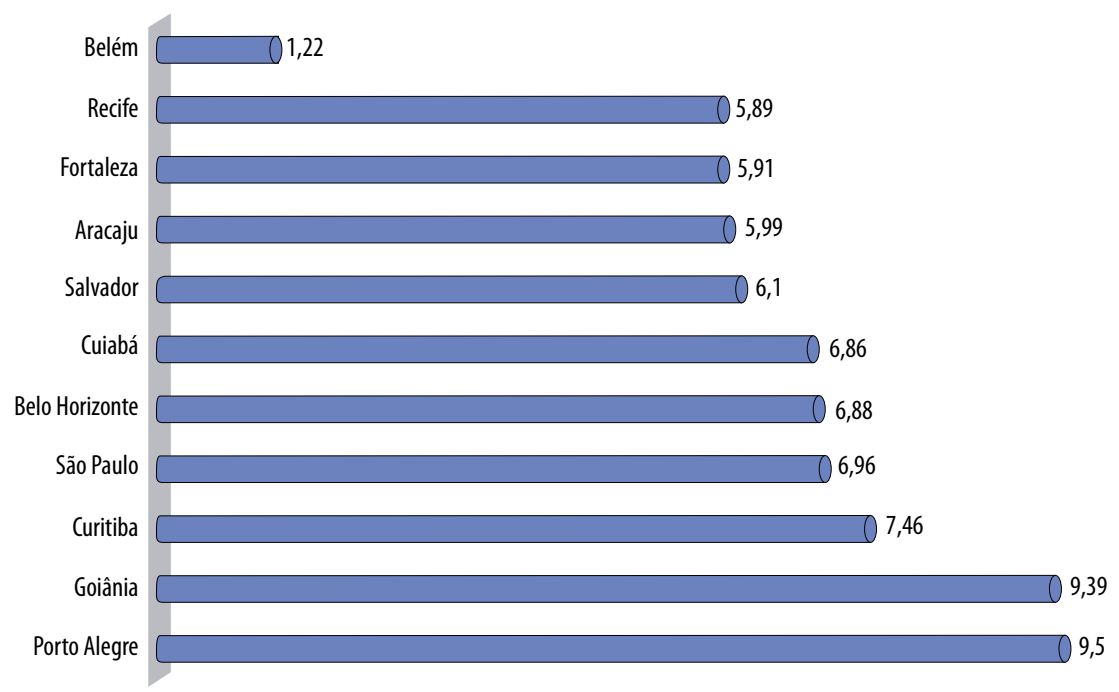

Figura 5 - Comparação entre as incidências de TIP em diferentes capitais (2000 a 2005). Fonte: Instituto Nacional de Câncer (INCA). 


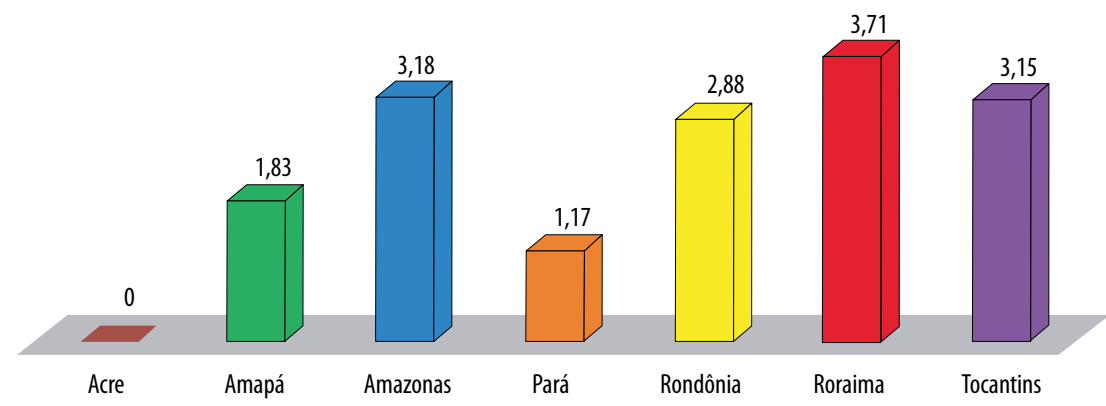

Figura 6 - Incidência estimada de neoplasias cerebrais para o ano de 2012 na região Norte. Fonte: Instituto Nacional de Câncer (INCA).

Analisando separadamente os subtipos histológicos que compõem a amostra de gliomas, observa-se que os glioblastomas constituíram $32 \%$ de todos os TIP e $45 \%$ do grupo de gliomas, seguidos por astrocitomas benignos (30\%), ependimomas $(9,5 \%)$, oligodendrogliomas (4\%) e gliomas mistos (2\%). Esses dados são congruentes com aqueles disponíveis pela IARC/OMS ${ }^{11}$ (International Association for Research on Cancer) em $2009^{2}$ e outros pesquisadores. ${ }^{2,6,7,31,32}$

O predomínio dos astrocitomas (84,5\%) na amostra é concordante com a literatura, sendo mais da metade (64\%) de natureza maligna - astrocitoma anaplásico (11\%) e glioblastoma (GBM) (53\%), ${ }^{4,6,7}$ também coincidindo com vários estudos clássicos sobre esse tema. ${ }^{2}$ Relevante acrescentar a existência de outro viés, dessa vez de seleção, ocasionado pela frequente necessidade de reintervenção cirúrgica nos pacientes submetidos a biópsias em outros hospitais do estado que prestam assistência ao SUS. Após o diagnóstico, os pacientes são transferidos para o HOL para o início do tratamento adjuvante, mas muitos deles são reavaliados com exames por imagem, detectando-se que foram submetidos, na verdade, a macrobiópsias, e necessitam de nova intervenção cirúrgica para controle da pressão intracraniana, citorredução e confirmação histopatológica.

$\mathrm{O}$ achado de $15 \%$ de meningiomas na amostra concorda com Gomes, ${ }^{33}$ discordando, no entanto, de outros autores, ${ }^{2,22,34-36}$ que consideram os meningiomas os tumores primários do SNC mais frequentes, seguidos pelos glioblastomas, o inverso do encontrado nos pacientes do HOL.

Quanto ao sexo, houve discreto predomínio do sexo masculino em nossa amostra (52\%), em concordância com os estudos de Argollo e Lessa ${ }^{21}$ e de Ohgaki, ${ }^{15}$ mas divergindo de Santos et al. ${ }^{20}$ da CBTRUS ${ }^{2}$ e de Gomes. ${ }^{31}$ A idade média dos pacientes encontrada foi de 36,5 , dado congruente com a literatura, ${ }^{1,2,5,18,29}$ que considera indivíduos entre a terceira e quinta década de vida como os mais acometidos pela doença, devido, em parte, pelo efeito acumulativo de mutações gênicas. ${ }^{21}$ Não houve diferença relevante entre as idades médias de acordo com os sexos.

\section{Conclusão}

Os resultados demonstram uma incidência de tumores intracranianos primários no estado do Pará da ordem de 1,22 para cada 100 mil pessoas, predominando gliomas (70\%), seguidos pelos meningiomas (15\%). Dentre os gliomas, como era de se esperar, astrocitomas foram os mais encontrados: $60,5 \%$ do total e $84,5 \%$ de todos os gliomas, com evidente predomínio dos astrocitomas graus III e IV, com $64 \%$, em relação aos astrocitomas graus I e II (36\%). Dentre astrocitomas malignos, glioblastomas foram o tipo histológico mais encontrado ( $32 \%$ dos TIP e $45 \%$ entre os gliomas). Ressaltamos as limitações inerentes aos estudos epidemiológicos na região Norte, com acesso desigual a recursos terapêuticos e diagnósticos e notificação deficiente, como principais fatores para certas divergências entre os resultados aqui encontrados e aqueles descritos pela literatura especializada no tema. Apesar disso, a elevada incidência de malignidade encontrada no estudo segue uma tendência observada na maioria dos serviços do mundo, onde os TIP em geral significam um quadro de neoplasias graves e de difícil resolução, razão pela qual estudos sobre o tema acrescentam informações que podem ser revertidas em estratégias de prevenção e intervenção por parte da equipe assistencial e pelas autoridades governamentais.

\section{Referências}

1. Samuels MA. Manual de Neurologia. $7^{\mathrm{a}}$ ed. Rio de Janeiro: Revinter; 2007.

2. CBTRUS Statistical Report: Primary Brain and Central Nervous System Tumors Diagnosed in the United States in 2004-2008. Source: Central Brain Tumor Registry of the United States, Hinsdale, IL, 2012. Disponível em: <http:/ www.cbtrus.org>.

3. IARC-WHO, órgão da Organização Mundial de Saúde (OMS). Disponível em: <http://wwwdep.iarc.fr/>. Acessado em: 30 ago. 2009. 
4. Schwartzbaum JA, Fisher JL, Aldape KD, Wrensch M. Epidemiology and molecular pathology of glioma. Nat Clin Pract Neurol. 2006;2(9):494-503.

5. Pereira ELR. Efeito do pisosterol em cultura de astrocitomas malignos [dissertação]. Belém: Universidade Federal do Pará; 2010.

6. Ministério da Saúde. Instituto Nacional de Câncer José Alencar Gomes da Silva. Estimativa 2012: incidência de câncer no Brasil/Instituto Nacional de Câncer José Alencar Gomes da Silva. Rio de Janeiro: INCA; 2011.

7. Kleihues P, Cavenee WK. Tumors of the central nervous system: pathology and genetics. Lyon, France: International Agency for Research on Cancer; 1997.

8. Abbas AK, Fausto N, Kumar V. Robbins \& Cotran. Patologia: bases patológicas das doenças. $8^{\mathrm{a}}$ ed. Rio de Janeiro: Elsevier; 2010.

9. Louis DN, Ohgaki H, Wiestler OD, Cavenee WK, Burger PC, Jouvet A, et al. The 2007 WHO classification of tumours of the central nervous system. Acta Neuropathol. 2007;114(2):97-109.

10. Piepmeier JM. Current concepts in the evaluation and management of WHO grade II gliomas. J Neurooncol. 2009;92(3):253-9.

11. Nakazato Y. [Revised WHO classification of brain tumours]. Brain Nerve. 2008;60(1):59-77.

12. Bauchet L. [Epidemiology and classification of gliomas]. Soins. 2009;(733):24-5.

13. DeAngelis LM. Brain tumors. N Engl J Med. 2001;344(2):11423.

14. DeMonte F, Gilbert MR, Mahajan A, McCutcheon IE editors. Tumors of the brain and spine. New York: Springer; 2007.

15. Ohgaki H. Epidemiology of brain tumors. Methods Mol Biol. 2009;472:323-42.

16. Brandes AA, Tosoni A, Franceschi E, Reni M, Gatta G, Vecht C. Glioblastoma in adults. Crit Rev Oncol Hematol. 2008;67(2):139-52.

17. Mao Y, Desmeules M, Semenciw RM, Hill G, Gaudette L, Wigle DT. Increasing brain cancer rates in Canada. CMAJ. 1991;145(12):1583-91.

18. Wen PY, Kesari S. Malignant gliomas in adults. N Engl J Med. 2008;359(5):492-507.

19. Lopez-Gonzalez MA, Sotelo J. Brain tumors in Mexico: characteristics and prognosis of glioblastoma. Surg Neurol. 2000;53(2):157-62.

20. Santos R, Frigeri L, Ordovás C, Frigeri M, Bertuol I, Guimarães CVA, et al. Epidemiologia dos tumores do sistema nervoso central, Hospital Nossa Senhora de Pompeia, Serviço de Neurocirurgia, a propósito de 100 casos estudados. Rev Cient AMECS. 2001;10(1):24-32.

21. Argollo N, Lessa I. Aspectos clínico-epidemiológicos das neoplasias cerebrais na faixa etária pediátrica no estado da Bahia, Brasil. Arq Neuropsiquiatr. 1999;57(2B):442-51.
22. GLOBOCAN. Worldwide Incidence and Mortality of Cancer, 2002 [computer program]. Lyon, France: IARC Press; 2002.

23. Parkin DM, Whelan SL, Ferlay J, Teppo L, Thomas DB. Cancer in Five Continents. Lyon, France: IARC Press; 2002.

24. McCarthy BJ, Schellinger KA, Propp JM, Kruchko C, Malmer B. A case for the worldwide collection of primary benign brain tumors. Neuroepidemiology. 2009;33(3):268-75.

25. Kohler BA, Ward E, McCarthy BJ, Schymura MJ, Ries LA, Eheman $C$, et al. Annual report to the nation on the status of cancer, 1975-2007, featuring tumors of the brain and other nervous system. J Natl Cancer Inst. 2011;103(9):714-36.

26. Cancer statistics registrations. Registrations of cancer diagnosed in 2008, England. Office for National Statistics, Ed.MB1 39.2010. Disponível em: <http://www. nationalarchives.gov.uk/>.

27. Schiff D, Wen PY. Cancer neurology in clinical practice. Totowa, NJ: Humana Press; 2003.

28. Rowland LP, Merritt HH. Tratado de Neurologia. $10^{\mathrm{a}}$ ed. Rio de Janeiro: Guanabara Koogan; 2002.

29. IBGE: Instituto Brasileiro de Geografia e Estatística. Disponível em: <http://www.censo2010.ibge.gov.br/ dados_divulgados. Acesso em: 18 abr. 2012.

30. Kallio M, Sankila R, Jääskeläinen J, Karjalainen S, Hakulinen T. A population-based study on the incidence and survival rates of 3857 glioma patients diagnosed from 1953 to 1984. Cancer. 1991;68(6):1394-400.

31. Gomes MM. Tumores primários do sistema nervoso central: aspectos epidemiológicos. Rev Bras Neurol. 1994;30(4):123-7.

32. Sanson M, Delattre JY. [Cerebral tumours in the adult. A real increase]. Rev Prat. 2006;56(16):1755-6.

33. Gomes VCC. Clínica e evolução dos tumores do sistema nervoso central na região da Beira Interior nos anos de 2007 e 2008 [dissertação]. Portugal: Covilhã. Universidade da Beira Interior; 2010.

34. Schoenberg BS, Christine BW, Whisnant JP. The descriptive epidemiology of primary intracranial neoplasms: the Connecticut experience. Am J Epidemiol. 1976;104(5):499510.

35. Instituto Português de Oncologia de Francisco Gentil. Registro Oncológico Nacional em 2001. Porto: Mediana; 2008.

36. Wöhrer A, Waldhör T, Heinzl H, Hackl M, Feichtinger J, Gruber-Mösenbacher $U$, et al. The Austrian Brain Tumour Registry: a cooperative way to establish a population-based brain tumour registry. J Neurooncol. 2009;95(3):401-11.

Endereço para correspondência

Edmundo Luís Rodrigues Pereira

Rua dos Pariquis, 1838, ap. 802, Jurunas

66033-590 - Belém, PA, Brasil

E-mail: eluis@ufpa.br 